EPJ Web of Conferences 79, 03010 (2014)

DOI: $10.1051 /$ epjconf/20147903010

(C) Owned by the authors, published by EDP Sciences, 2014

\title{
Direct energy conversion using liquid metals
}

\author{
Alexandru Onea, Nerea Diez de los Rios Ramos, Wolfgang Heringa ${ }^{\text {, }}$ Robert Stieglitz \\ and Peter Moster \\ Karlsruhe Institute of Technology, Hermann-von-Helmholtz-Platz 1, \\ 76344 Eggenstein-Leopoldshafen, Germany
}

\begin{abstract}
Liquid metals have excellent properties to be used as heat transport fluids due to their high thermal conductivity and their wide applicable temperature range. The latter issue can be used to go beyond limitations of existing thermal solar energy systems. Furthermore, the direct energy converter Alkali Metal Thermo Electric Converter (AMTEC) can be used to make intangible areas of energy conversion suitable for a wide range of applications. One objective is to investigate AMTEC as a complementary cycle for the next generation of concentrating solar power (CSP) systems. The experimental research taking place in the Karlsruhe Institute of Technology (KIT) is focused on the construction of a flexible AMTEC test facility, development, test and improvement of liquid-anode and vapor-anode AMTEC devices as well as the coupling of the AMTEC cold side to the heat storage tank proposed for the CSP system. Within this project, the investigations foreseen will focus on the analyses of BASE-metal interface, electrode materials and deposition techniques, corrosion and erosion of materials brought in contact with high temperature sodium. This prototype demonstrator is planned to be integrated in the KArlsruhe SOdium LAboratory (KASOLA), a flexible closed mid-size sodium loop, completely in-house designed, presently under construction at the Institute for Neutron Physics and Reactor Technology (INR) within KIT.
\end{abstract}

\section{Introduction}

In the frame of the changes in the German energy policy, new technologies are under investigation that can provide clean, efficient, and economically viable renewable energies. In this context, AMTEC devices offer promising performances since they are able to convert heat directly into electrical energy without generating any toxic or greenhouse by-products and can achieve theoretically high conversion efficiency. Since AMTEC cells can be coupled to any heat source that can provide a temperature in the range of $600-1000^{\circ} \mathrm{C}$, AMTEC can be considered as a very promising technology with a large spectrum of different possible applications, e.g. in the field of renewable energies.

At INR, AMTEC technology has been developed as a spin-off from the liquid metal technology in the nuclear sector. The background existing at INR allowed us to consider the AMTEC converter for new research activities and as a complementary system of a liquid metal based solar power plant for high temperature. This new concept of thermal solar power plant profits from a compact heat storage tank. For a detailed description of the combined AMTEC-CSP cycle we refer to the paper [1]. The

\footnotetext{
${ }^{\text {a }}$ Corresponding author: wolfgang.hering@kit.edu
}

This is an Open Access article distributed under the terms of the Creative Commons Attribution License 4.0, which permits unrestricted use, distribution, and reproduction in any medium, provided the original work is properly cited. 
major advantages of this design are the increased efficiency (estimated at about 30\%) in comparison to conventional solar power plants (15-25\% efficiency), the best utilization of the stored thermal energy, and a longer and more flexible operability. On the other hand, the estimated investing costs are higher and further research and development investigations are needed. Another combination of a CSP and AMTEC was studied also in [2], where it is reported that the total efficiency of the combined system could reach $20 \%$.

\section{Overview of direct energy conversion systems for electricity production}

In the frame of the direct energy conversion systems for electricity production using liquid metals, without considering all available systems, one can identify the main fields as being thermoelectric power generation (Seebeck effect, AMTEC cells), thermionic power generation, electrochemistry (fuel cells, Redox cells) and magnetohydrodynamics.

Within the direct energy conversion systems are the thermoelectrics, which can be divided into converters that use the Seebeck effect and AMTEC converters, thermionics, solar cells and fuel cells. Thermoelectric converters functioning based on the Seebeck effect have achieved efficiencies of $\sim 15 \%$. A good thermoelectric converter needs to reach a low thermal conductivity while in the same time the electrical conductivity remains high; these two requirements must be accomplished in order to reach the required high operational temperatures. State of the art in this energy conversion system brings one to the nanoscale tailoring technique, in order to get closer to the objectives of a good thermoelectric converter [3]. Despite of the progress in this technique, there is still the need of finding more appropriate materials with a higher value of the figure of merit $\mathrm{ZT}=\mathrm{S} 2 \sigma \mathrm{T} / \kappa$, where $\mathrm{S}$ denotes Seebeck coefficient, $\sigma(\Omega-1 \mathrm{~m}-1)$ represents the electrical conductivity, T is temperature and $\kappa(\mathrm{WK}-1 \mathrm{~m}-1)$ denotes the thermal conductivity.

AMTEC has a theoretical efficiency close to that of the Carnot cycle, namely a value of $45 \%$ can be theoretically obtained. Further, AMTEC devices are flexible regarding the input heat source, making therefore this thermoelectric converter a promising energy conversion system.

Previous investigations of AMTEC devices performed at KIT report an efficiency of up to 25\% [4]. The longest known operating time of AMTEC, reported in [5], is more than 5 years. Still, the power loss occurring during long time operation is one of the main issues of AMTEC that requires further investigations, as well as the technical challenges such as high temperature operation, ceramic-metal interface and electrical issues like electrodes or current collector.

The thermionic converters have achieved efficiencies of $\sim 20 \%$. Although nanoparticles are being investigated for an improvement of the material characteristics, the issues that still remain to be solved are the difficulties associated to the high operating temperature, which are larger than $1500{ }^{\circ} \mathrm{C}$, the complicated construction and the associated high costs.

Fuel cells however have a much higher efficiency ranging from 35 up to $60 \%$ [6]. In the last years a significant progress has been achieved regarding operating temperature, vessel technologies, costs reduction, efficiency. Nevertheless, issues such as the fuel storage, fuel generation, fuel infrastructure for vehicles, fuel gases, lifetime, and high costs are still open and need to be further improved.

For the sake of comparison, the researches in the solar cells lead to an enhancement of the efficiency. Actual laboratory efficiencies achieved for crystalline silicon solar cells go up to $24.4 \%$, while the record in amorphous silicon solar cells reaches 15\% [7]. All single-junction cells have a theoretical maximal efficiency value of $31 \%$. On the other hand, multi-junction (or tandem) solar cells have a theoretical efficiency of $47.5 \%$ for a triple-junction. In the industrial production, the highest efficiency achieved is $31 \%$ for silicon nanowires. Nevertheless, the photovoltaic technology (in particular the multi-junction cells) is known to have expensive production costs. Other issues still to be solved are the contents of toxic elements or rare elements and the high "environmental" price [8]. 
Table 1. Comparison of temperature, efficiency and most significant issues for different direct energy conversion systems.

\begin{tabular}{|l|c|c|l|}
\hline $\begin{array}{l}\text { Energy conversion } \\
\text { system }\end{array}$ & $\operatorname{Tmin} / \operatorname{Tmax}\left[{ }^{\circ} \mathrm{C}\right]$ & $\eta[\%]$ & Issues \\
\hline $\begin{array}{l}\text { Thermoelectric converter } \\
\text { (Seebeck effect) }\end{array}$ & $100 / 900$ & $<20$ & $\begin{array}{l}\text { Availability of good semiconductors } \\
\text { that sustain high temperatures }\end{array}$ \\
\hline AMTEC & $200 / 1000$ & $\sim 20(40)$ & Lifetime and efficiency under research \\
\hline Thermoionic converter & $\operatorname{Tmax}>1500$ & $<20$ & High operating temperatures \\
\hline Solar cell & $30 / 60$ & $15-31$ & $\begin{array}{l}\text { High costs, "environmentally" } \\
\text { expensive, contain toxic elements }\end{array}$ \\
\hline Fuel cell & $200 / 1000$ & $35-60$ & $\begin{array}{l}\text { Fuel storage, fuel generation and } \\
\text { lifetime research needed. High costs. }\end{array}$ \\
\hline
\end{tabular}

All mentioned direct energy conversion systems require further development, despite significant research progress. The fuel cells have reached the most advanced technological level, with industrial production already started, e.g. in the automotive sector. In the field of renewable energies, AMTEC technology has the potential to be an important contributor especially when coupled to a clean heat source that does not emit $\mathrm{CO}_{2}$ gases.

Table 1 summarizes the characteristics of the direct energy conversion systems mentioned above.

\section{Alkali Metal Thermal to Electric Converter (AMTEC)}

At the Karlsruhe Research Center (formerly FZK, nowadays KIT) the experimental investigation of AMTEC cells was started in 1988 taking advantage of the knowledge of using sodium in the nuclear field. Several prototypes, both liquid and vapor-anode, were built and analyzed until 2001. The investigations were focused on testing electrodes, current collectors, BASE-metal interfaces as well as conducting long time stability tests [4].

\subsection{Operating principle}

The Alkali Metal Thermal Energy Converter (AMTEC) is a thermally regenerative electrochemical device for direct conversion of heat into electricity. The fundamental component of the device is a $\beta$ "-alumina solid electrolyte (BASE) ceramic, which has a large ionic conductivity and a negligible electronic conductivity. The process of ionization of sodium occurs at the surface of BASE, where the sodium is dissociated into $\mathrm{Na}^{+}$that diffuse through BASE and electrons that are redirected by a voltage gradient through an electric load to produce electricity. Among several versions of BASE, the B"-alumina solid electrolyte presented the most attractive properties regarding ionic resistance.

The pressure difference applied across the BASE represents the driving force for the $\mathrm{Na}^{+}$across the ceramic. An AMTEC cell is divided by BASE into a high temperature $\left(600-1000^{\circ} \mathrm{C}\right)$, high pressure $\left(0.5 \times 10^{5}-2 \times 10^{5} \mathrm{~Pa}\right)$ region and a low temperature $\left(150-500^{\circ} \mathrm{C}\right)$, low pressure region $(<100 \mathrm{~Pa})$, as presented in Fig. 1. In a liquid anode cell, the liquid itself serves to transport the electrons towards the electrical load, while the porous electrode (typical thickness of $1-5 \mu \mathrm{m}$ ) on the cathode side serves for the recombination of the sodium ions with the electrons. In a vapor anode cell, porous electrodes are placed on both sides of BASE, to capture the electrons and circulate them through current leads towards and from the external load.

Sodium ions recombine with electrons on the cathode side to form neutral sodium molecules that will condensate on the cooled surface of a condenser forming liquid sodium. Further, an electromagnetic pump or a wick can be employed to circulate back the liquid sodium to the hot side, so that the cycle can be repeated. 


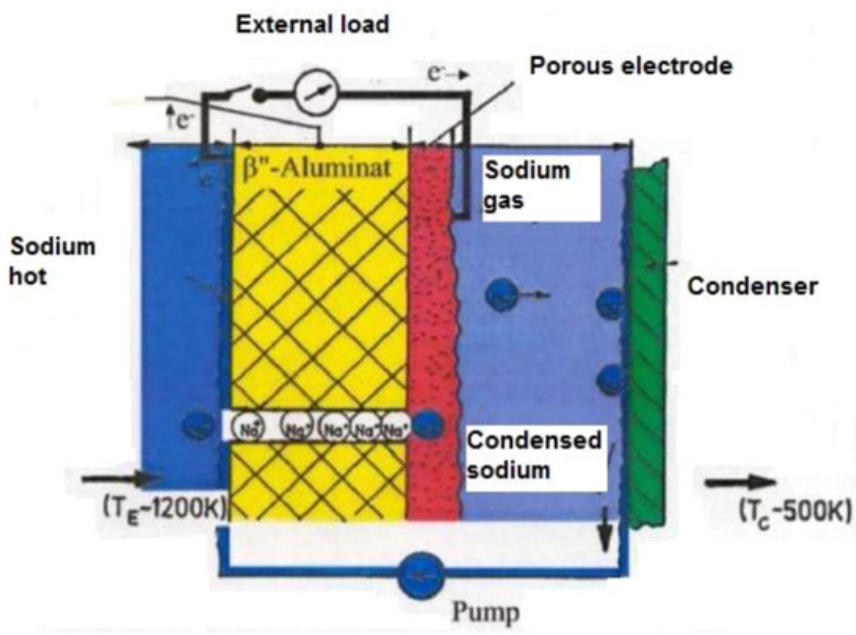

Figure 1. Schematic of the AMTEC operational principle.

The concept of an AMTEC cell was patented by Kummer [9] in 1969, based on the sodium-sulphur technology. The first prototypes employed liquid sodium on the anode side, and since 1990s vapor-vapor cells were developed with the aim of increasing the voltage and the efficiency. Several liquid-anode and vapor-anode cells were developed and investigated at INR for more than 10 years, providing a significant knowledge that is presently used for further studies.

Three alkali metals (sodium, potassium and lithium) are known to be considered as working fluids for AMTEC cells. Most cells employed sodium due to the availability of the electrolyte. Potassium offers interesting advantages in comparison with sodium: lower latent heat of vaporization, lower specific heat capacity and larger vapor pressure. These properties lead to a higher conversion efficiency of the K-AMTEC cell in comparison with Na-AMTECs, for the same operating conditions. However, the disadvantages of the K-AMTECs are the increased ionic resistivity in the electrolyte, which leads to larger electrical losses, the larger molecular weight, which leads to larger flow rates for the same current, and reduced operating experience. An overview of the influence of these fluids on the performances of the cell is summarized in [2]. Due to its low vapor pressure lithium cells require larger operating temperatures. Nevertheless, a lithium operating cell is reported to be investigated in [9], where a lithium oxide electrolyte that proved stable performances was employed.

\subsection{Pending issues}

The AMTEC technology has many interesting advantages, such as a large efficiency (up to $40 \%$ for robust designs), flexibility regarding the heat source, large power density, estimated up to $0.5 \mathrm{~kW} / \mathrm{kg}$ in [11], suitability for modular design and further use of the exhaust heat, virtually maintenance-free due to the absence of moving parts, noiseless and compact. Another major advantage of this technology is the estimated low production costs, making the cell fabrication economical.

However, AMTEC cells face two major drawbacks, namely the significant reduction of the electrical power on long time operation and the reduced conversion efficiency (compared to the theoretical value).

A power output degradation of about $50 \%$ after 2 years operation is reported in [12]. Such power losses appear to be typical for AMTEC cells and are reported by several research groups. In surprising and encouraging contrast to this issue, the longest operation period up to now for a small, multi-tube AMTEC converter of more than 5 years, for which no indications of power loss appeared is reported 
in [5]. Although no clear suggestions are given that can explain this positive achievement and taking into account the fact that the technology used appears to be rather conventional (stainless steel shell, titanium nitride TiN electrodes), it seems that the good thermal insulation employed prohibits the heat losses and can potentially explain the lack of the power loss. Nevertheless, the issue of power degradation remains further under investigation.

The BASE is the main source of power degradation, estimated up to $76 \%$ from the total loss, while the losses in the electrode can reach up to $20 \%$, as estimated in [13]. The sources of power degradation can be divided into issues related to the BASE, to the electrode and issues related to sodium. The issues related to BASE are the loss of sodium, the appearance of cracks, the formation of molten dendrites and grain growth. These processes lead to either reduced ionization of sodium or to increased ionic resistance. The main reason for the degradation caused by electrodes is the grain growth effect, which leads to the decrease of the total number of grains and therefore to the decrease of the total contact area between BASE and electrode, consequently reducing the exchange current. In [14] is reported a model for the estimation of the electrode lifetime, based on the grain growth mechanism. The issues related to sodium are the chemical reactions that can occur due to the demanding operating conditions (large temperatures and pressures), which lead to chemical products that can deposit or enter the BASE, thus negatively affecting the ionic conductivity.

\section{AMTEC projects and test facility}

\subsection{LIMTECH and HEMCP projects}

Presently several experiments are foreseen and will be performed in the frame of two research programs, namely in the frame of the Helmholtz alliance on liquid metal technology (LIMTECH project - http: //www .hzdr.de/db/Cms?pOid=36929\&pNid=2920) and Helmholtz Energy Materials Characterization Platform (HEMCP project).

The main aims in the frame of the LIMTECH project can be summarized as:

- Construction of an AMTEC test facility and of a demonstrator;

- Experimental test campaign for the analysis of the AMTEC cell performances (thermo-dynamical and electrical), degradation behavior and lifetime prediction;

- Identification of development directions for AMTEC design optimization;

- Development and construction of advanced design liquid-anode and vapor-anode cells;

- Investigation of a modular design for AMTEC cells.

In the frame of the HEMCP program, the research will take place in collaboration with different institutes at KIT and will be focused on material characterization (analysis of the BASE stability, BASE-metal interface, corrosion/erosion, material creep behavior, analysis of materials for electrodes) in liquid sodium, and characterization of the material deterioration mechanisms. The investigation of the electrode deposition techniques is also envisaged. For these purposes a material test facility is under development and construction at INR. The facility is designed to sustain temperatures up to $1000 \mathrm{~K}$ in the test section. In order to investigate the behavior of materials under such extreme conditions both constant operation and cyclic tests are foreseen, as well as long time tests. This spectrum of investigations will also allow concluding about the AMTEC lifetime.

\subsection{AMTEC test facility - ATEFA}

The first aims of our investigation are to regain the knowledge available at INR and to construct a prototype that can demonstrate the functionality of this technology. Further, this demonstrator will be 


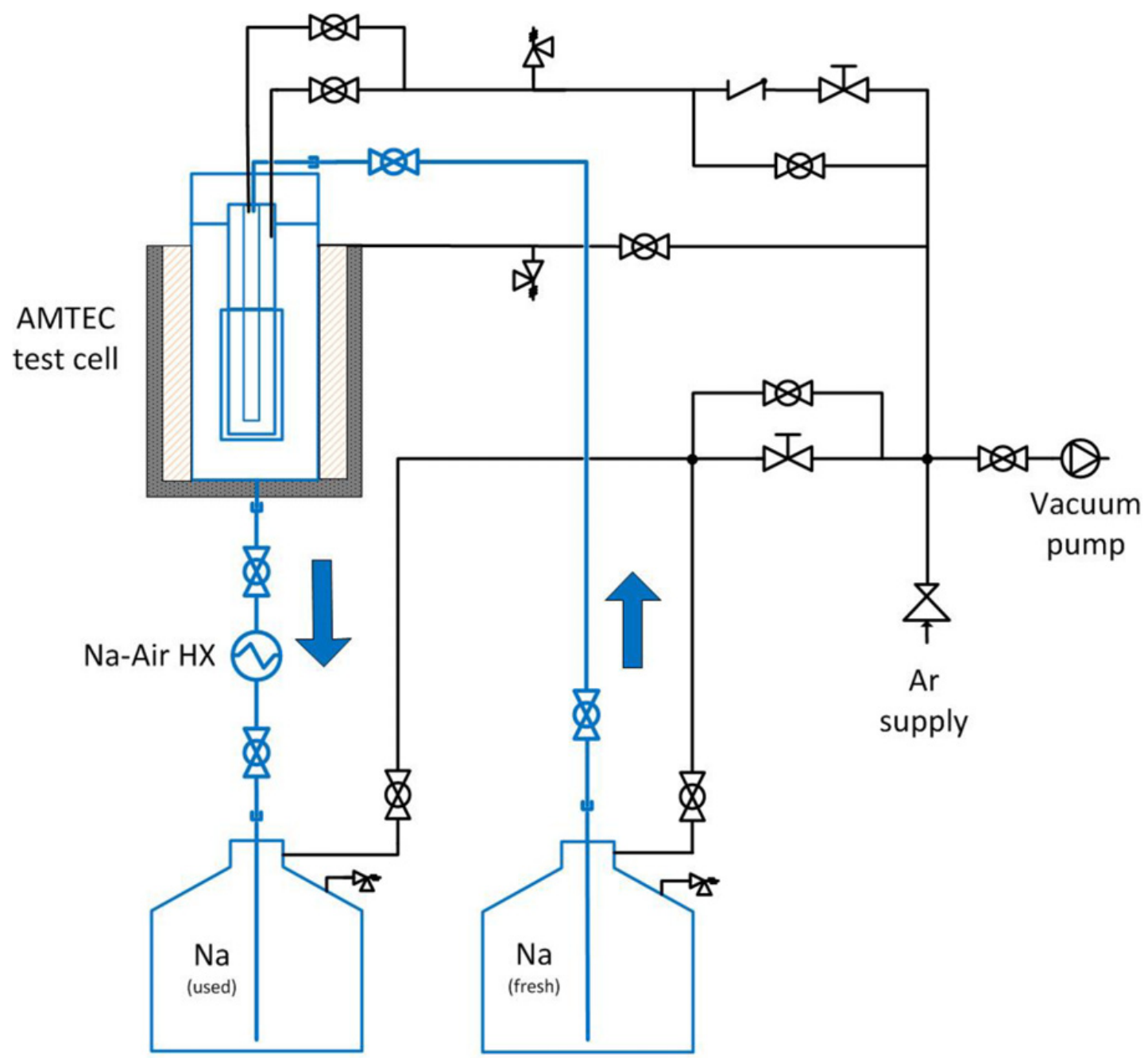

Figure 2. Schematic diagram of the ATEFA facility at KIT (blue arrows denote Na flow, black lines - Ar side).

used to gain operating experience and as a starting point for further design optimization and experimental tests foreseen in the projects LIMTECH and HEMCP.

The AMTEC TEst FAcility (ATEFA), presented in Fig. 2, is an open system that contains two sides, namely sodium and argon. The sodium flow is controlled and driven by the pressure applied on the argon side, which is used to "push" the sodium in the facility. Argon gas is used due to its known inert behavior to sodium and to prevent sodium oxidation. The test cell of the facility is designated to be flexible, to allow the investigation of several AMTEC prototypes. Temperatures up to $1000^{\circ} \mathrm{C}$ and pressures up to 2 bars can be reached in the test cell, requiring special care in the design.

The sodium side (blue lines in Fig. 2) contains two storage tanks, foreseen for fresh and "used" sodium, the test cell and the sodium-air heat exchanger. On the argon side (black lines in Fig. 2) a mass flow controller is foreseen to regulate the sodium flow rate and a pressure controller is used to hold the pressure constant in the cell. The facility is cleaned and drained by employing a vacuum pump. The 


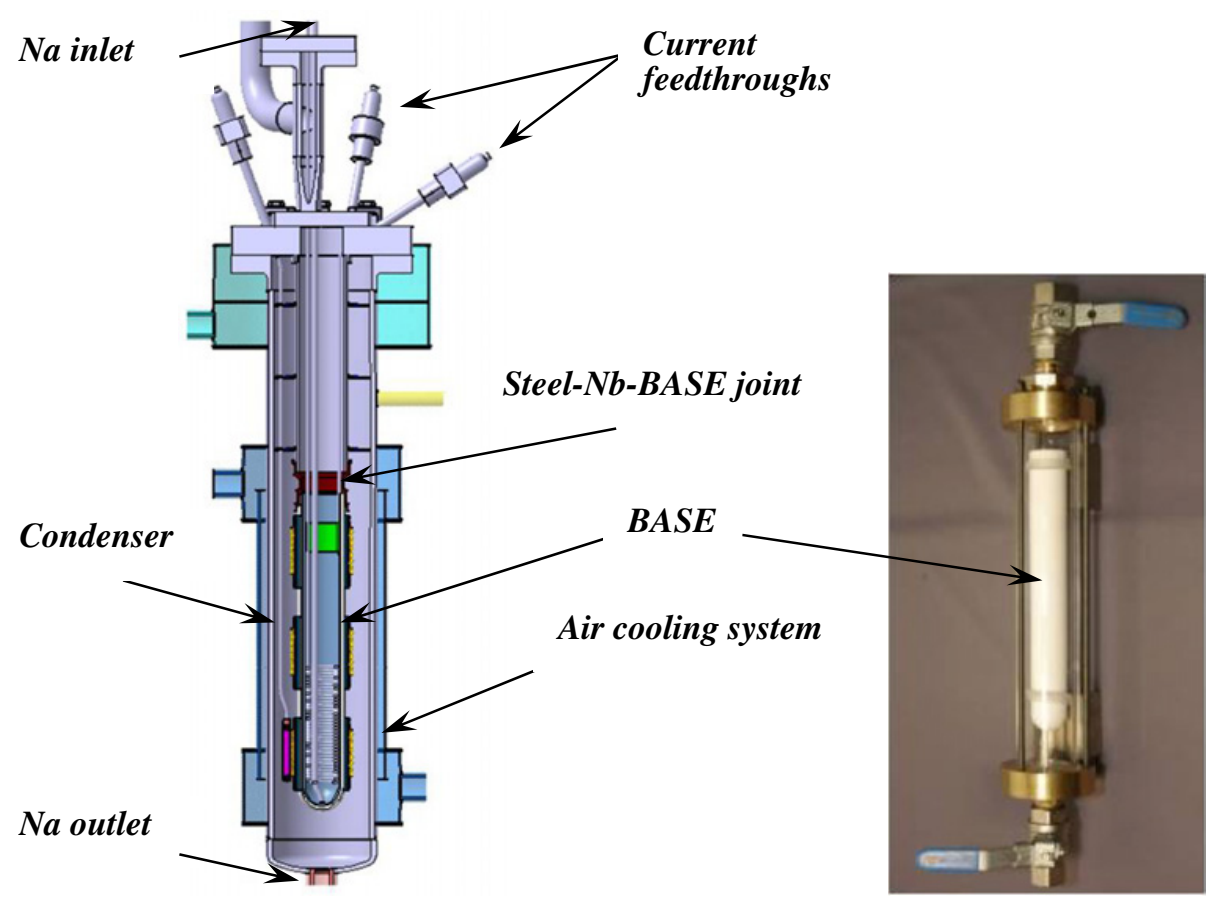

Figure 3. AMTEC test cell (left side) and BASE finger (right side).

argon side is split in the supply branch and the pressure branch; namely the aim of the first branch is to deliver the sodium to the test cell, while the task of the second branch is to maintain the pressure in the test cell during operation. For safety reasons, two safety valves are foreseen on each argon branch to prevent the built-up of pressure.

The layout of the facility is based on a safety oriented design, relying on the international knowledge in handling sodium and on our experience. First of all, the usage of water has been eliminated from the design phase. The sodium amount available in the facility is rather small ( $\sim 4$ liter), reducing therefore the damage in case of an incident. For safety reasons, the high temperature needed for experiments has been constrained only to the test cell, while the rest of the facility is held at low temperature, namely $\sim 150^{\circ} \mathrm{C}$ in the sodium supply branch. All valves of the facility are manually operated and have extensions if placed in a high temperature part of the facility. Another safety measure is that the entire facility is housed in a thermally isolated metallic box that can retain the sodium in case of a leakage. The box is used also as a thermal insulator.

The AMTEC test cell and the finger-type BASE encapsulated in a mobile configuration are shown in Fig. 3. Considering the sodium mass flow rate for one BASE element [15] as $\dot{m}=M I / F$, where $M=23 \mathrm{~g} /$ mole represents the sodium molar mass, $F=96485$ (As)/mole the Faraday's constant and $I$ the electrical current, the thermal output of the cell for typical operation conditions can be estimated to be $\dot{Q}_{\mathrm{Na}}=\dot{m}_{\mathrm{Na}} c_{p N a}\left(T_{\text {in }}^{\mathrm{Na}}-T_{\text {out }}^{\mathrm{Na}}\right) \sim 25 \mathrm{~W}$, where $c_{p}$ represents the heat capacity and $T$ the temperature.

The BASE is placed in the central part and it is connected with a niobium $(\mathrm{Nb})$ joint to a steel flexible cylinder attached to the upper holding flange. The choice of $\mathrm{Nb}$ is related to the fact that $\mathrm{Nb}$ has a similar thermal expansion coefficient as the BASE. The Nb joint is brazed to both BASE and steel. Other interfaces and connections are planned to be investigated in the future. The outer side of the cell serves as condenser and it is being cooled with air. The test cell size allows testing several BASE ceramics having different geometrical sizes. Since the length of the upper steel cylinder and of the BASE 
can be varied, the effect of the gap between the condenser and the BASE can also be investigated. To heat up the sodium a heater is considered at the bottom inner side of the BASE. By this solution the high temperature region is restricted only to a limited area, reducing therefore the thermal stresses on the materials.

For the electrodes several materials are to be considered, such as molybdenum (Mo), titanium nitride (TiN) or rhodium tungsten (RhW). The electrode deposition will be performed at KIT in the frame of the HEMCP project.

Between the cell and the storage tank a sodium-air heat exchanger is foreseen to cool further the sodium prior to its entrance in the "cold" storage tank. Our first estimations indicate that sodium can be cooled about $50-100{ }^{\circ} \mathrm{C}$, function on the air flow rate. The volume of one tank has been restricted to about 4.5 liter, so that it can be manually handled.

The measurements presently foreseen will focus on the performance of the cell (voltage, current intensity, internal resistance) as a function of the operating temperature. Further, the temperature distribution in the cell and the vapor pressure between the BASE and the condenser are also monitored.

The design of the facility is finished and it is now in the construction phase, with the first operational tests foreseen for March 2014.

\section{Summary and outlook}

The final design of a test facility to be used for experimental investigations of AMTEC converters is reported in this paper. The safety oriented design of the facility relies on the international knowledge in handling sodium and our experience. The design of the facility aimed to provide a rather simple, yet robust construction that can be used to gain operational experience with AMTEC devices and prove the functionality of this technology. The work is already financially supported in the frame of the projects LIMTECH and HEMCP. The facility is presently under construction and the start of the AMTEC experimental campaign is foreseen for the first half of 2014.

\section{References}

[1] Hering, W., Stieglitz, R., Wetzel, R. Application of liquid metals for solar energy systems. 2nd Eur. Energy Conf., Maastricht (2012)

[2] Wu, S.-Y., Xiao, L., Cao, Y., Li, Y.-R. A parabolic dish/AMTEC solar thermal power system and its performance evaluation. Applied Energy 87, 452-462 (2010)

[3] Tervo, J., Manninen, A., Ilola, R., Hänninen, H. State-of-the-art of thermoelectric materials processing, VTT-WORK-124 (2009)

[4] Heinzel, V., Huber, F., Peppler, W., Will, H. Thechnological tests with respect to the use of beta alumina in Alkali-Metal-Thermo-Electric Converters (AMTEC). Key Eng. Mat. 59\&60, 381-394 (1991)

[5] Hunt, T. K., Rasmussen, J. R. Fractional Watt AMTEC Converter. Space Techn. Applications Int. Forum - STAIF 2006, 567-572 (2006)

[6] Elmer, T., Riffat, S. B. State of the art review: fuel cell technologies in the domestic built environment. Int. Conf. on Sustainable Energy Techn., Canada (2012)

[7] Dimova-Malinovska, D. The state-of-the-art and future development of the photovoltaic technologies - the route from crystalline to nanostructured and new emerging materials. J. Physics. Conference Series 253 (2010)

[8] Miles, R. W., Hynes, K. M., Forbes, I. Photovoltaic solar cells: An overview of stato-of-the-art cell development and environmental issues. Pr. in Crystal Growth and Ch. Mat., 51, 1-42 (2005)

[9] Kummer, J. T., Weber, N. Forbes, I. Thermo-electric generator. U.S. Patent No. 3458356 (1969) 
$3^{\text {rd }}$ European Energy Conference

[10] Lysenko, G. P. Lithium AMTEC with gas-diffusion cathode, Energy Conversion Eng. Conf., IECEC 96, 2, 890-893 (1996)

[11] Cole, T. Thermoelectric Energy Conversion with Solid Electrolytes. Science 221, 915-920 (1983)

[12] Merill, J. Schuller, M., Huang, M. Vacuum testing of high-efficiency multi-tube AMTEC cells: February 1997-October 1997, Space Tech. and Applications Int. Forum, 420, 1613-1620 (1998)

[13] Lodhi, M.A.K., Vijayaraghavan, P., Daloglu, A. Time-dependent BASE performance and power degradation in AMTEC, J. Power Sources 93, 41-49 (2001)

[14] Ryan, M.A., Kisor, A., Williams, R F., Jeffries-Nakamura, B., O'Connor, D. Lifetimes of Electrodes for AMTEC Cells, 29th Intersociety Energy Conversion Eng. Conf., 877-881 (1994)

[15] Tournier, J.-M., El-Genk, M. S., Design optimization of high-power, liquid anode AMTEC, Space Techn. Applications Int. Forum - STAIF 2003, 740-750 (2003) 